\title{
Gelsemium sempervirens and animal behavioral models
}

\section{Paolo Bellavite*}

Department of Pathology and Diagnostics, University of Verona, Verona, Italy

*Correspondence: paolo.bellavite@univr.it

\section{A commentary on}

Gelsemine and Gelsemium sempervirens L. extracts in animal behavioral test: comments and related biases

by Chirumbolo, S. (2011). Front. Neur. 2:31. doi: 10.3389/fneur.2011.00031

In traditional Materia Medica, Gelsemium sempervirens ( $G$. sempervirens) is described as a remedy for a variety of anxiety-like psychological and behavioral symptoms, however consistent evidence of its efficacy is lacking. In our recent experimental studies (Bellavite et al., 2009; Magnani et al., 2010), groups of CD1 mice were treated with highly diluted solutions of G. sempervirens, or with conventional anxiolytic drugs, or with a placebo consisting of only the solvent (control groups). The results showed that G. sempervirens reduced anxiety and fear in a manner quantitatively comparable to the effects of normal anxiolytic drugs, but without provoking any sedation side-effects (which instead did occur with buspirone in that experimental set-up). These findings are consistent with those obtained by a group at the University of Strasbourg, which found that the same plant compound had a direct effect on the production of allopregnanolone by rat neurons grown in cell culture plates (Venard et al., 2009). Thus it is likely that the mechanism involved is the regulation of production of endogenous neurosteroids through glycinergic receptors. The chief innovation of this line of research lies in the fact that, until now, natural remedies based on G. sempervirens had been used only on humans, but without consistent evidence of efficacy or with controversial results. Since these remedies also appear to work on animal models and on in vitro systems, this indicates that they do not have merely a "placebo" effect, and that this type of pharmacology using ultra low doses - referred also as "nanopharmacology" - merits further investigation and validation.
The "commentary" entitled "Gelsemine and G. sempervirens L. extracts in animal behavioral test: comments and related biases" (Chirumbolo, 2011) is explicitly announced as a commentary on a recent paper from our research group (Magnani et al., 2010). The commentary does not make clear which are the contested points, nor the reason for the imputation of "related biases" in the title of the letter. The author maintains that "no pharmacological studies evaluated anti-anxiety activity of Gelsemium prior to the recently reported evidence of Dutt et al. (2010)" In point of fact, our first results on this subject were published in 2009 (Bellavite et al., 2009).

In the first part of the text, the author dwells at length upon the toxicity of the plant and of one of its active principles, gelsemine. These facts are well-known in the literature, but have no bearing on the question of the pharmacological effect on animal behavior, which in the research addressed by this commentary (Magnani et al., 2010), and in others cited therein (Venard et al., 2008, 2009), is accomplished through the administration of extremely low doses that are expected to be non-toxic. According to literature, in emergencies due to Gelsemium poisoning, gelsemine was sustained as the main marker of toxicity and was detected in the urine at concentrations ranging from 10 to $120 \mathrm{ng} /$ $\mathrm{ml}$ (Lai and Chan, 2009); as a matter of facts, in our studies, the maximum concentration of gelsemine in the drug samples tested (precisely in the fourth centesimal dilution from mother tincture) was $0.0021 \mathrm{ng} / \mathrm{ml}$ and we administered $0.3 \mathrm{ml}$ to $30 \mathrm{~g}$ weighing mice, with a further dilution of about 100-fold assuming uniform distribution. In any case, since no clinical data about potential adverse effects of G. sempervirens for chronic use in humans are available, the safety issue needs to be better addressed.

The commentary correctly reports that our work has demonstrated anxiolytic effects of high dilutions of G. sempervirens using two behavioral tests, the open field (OF) and light-dark (LD) tests, but then notes that "effects in the elevated plusmaze, an assay considered to be important in behavioral assessment of potential anxiolytic compounds, have not been assessed." The author of the commentary should know that there exist dozens of tests for animal behavior, out of which each protocol employs, for theoretical and practical reasons, whichever ones the experimenter deems best suited for the purposes of the research. OF (Prut and Belzung, 2003) and LD (Bourin and Hascoet, 2003) are two validated animal models, which allow to acquire various behavioral parameters and are widely employed in neuropsychopharmacology for drug screening (Belzung and Griebel, 2001; Bourin et al., 2007). Classic anxiolytics (benzodiazepines) as well as the newer anxiolytic-like compounds (e.g., serotonergic drugs) and natural compounds (Chen et al., 2004) have been tested using these paradigms. Therefore, the five different behavioral variables extracted from $\mathrm{OF}$ and LD in our investigation are sufficient and demonstrative enough of the psychopharmacological effect, although in the view of the results of our study it is not unreasonable to envisage the use of additional complementary tests for future studies on this topic.

Immediately afterward in the commentary we read that "Actually, the physical integration of different current tests in one single apparatus, should contribute to increased reliability, rapidity, and comprehensiveness in behavioral testing." This might at first glance seem a good idea; but in reality, every researcher in the field of experimental psychopharmacology knows that each model must be validated and is designed to evaluate different parameters. No-one has ever dreamed of using an apparatus which "physically integrates" OF, LD, and the elevated plus-maze.

The crux of the matter is reached when the author maintains that "criticism may be raised about biases in their experimental context," citing the works of Venard (Venard et al., 2008, 2009) and of our own group 
(Magnani et al., 2010). The author dwells at length on the effects of the active principle gelsemine, but puts forward no arguments in support of his claim of possible "bias" in the research, so that this assertion is impossible to discuss or controvert. In the central paragraph of the commentary, we find a treatise on the "placebo" and "nocebo" effects (all well-known phenomena in pharmacology), but not applicable to the work in question, which adopted rigorous double blind controls, consisting of the same solvent as the drug but without the active principle, precisely to rule out these types of effects. The net effect of the drug was always calculated as a percentage relative to the behavioral parameter measured in the mice treated with placebo. Indeed in the discussed study appropriate blinding, dummy medication, positive controls, and similar testing environment for all conditions were used. These measures are be sufficient to minimize placebo/nocebo biases.

Finally, the author implies - without demonstrating it - that there might be a bias in the statistics, where he maintains that "according to some Authors, the statistical power of behavioral studies to detect relationships is quite low: the power to detect a medium effect is $39-47 \%$ and in this case only $10-20 \%$ of tests exceeded the recommended minimum criterion of $80 \%$, as assessed by Cohen and colleagues." This assertion is in general terms highly debatable, and by no means endorsed by researchers in the field of behavioral psychopharmacology. With respect to our work, it is false (most experiments gave positive results and the ANOVA was highly significant) and ungrounded because it is impossible for Chirumbolo to have computed the $10-20 \%$ percent of tests from our data which are supplied as population averages. Moreover, in our work we clearly specify that the power of the study needed to achieve statistical certainty was correctly computed before undertaking the trials.

We obviously agree with Chirumbolo's conclusion that "Certainly, research on the anti-anxiety potential of Gelsemium alkaloids needs further investigative efforts, in order to comprehend the role of this plant in neuropharmacology," and await other experimental contributions, however it certainly does not constitute a criticism of our work.

\section{REFERENCES}

Bellavite, P., Magnani, P., Marzotto, M., and Conforti, A. (2009). Assays of homeopathic remedies in rodent behavioural and psychopathological models. Homeopathy 98, 208-227.

Belzung, C., and Griebel, G. (2001). Measuring normal and pathological anxiety-like behaviour in mice: a review. Behav. Brain Res. 125, 141-149.

Bourin, M., and Hascoet, M. (2003). The mouse light/ dark box test. Eur. J. Pharmacol. 463, 55-65.

Bourin, M., Petit-Demouliere, B., Dhonnchadha, B. N., and Hascoet, M. (2007). Animal models of anxiety in mice. Fundam. Clin. Pharmacol. 21, 567-574.

Chen, S. W., Min, L., Li, W. J., Kong, W. X., Li, J. F., and Zhang,Y. J. (2004). The effects of angelica essential oil in three murine tests of anxiety. Pharmacol. Biochem. Behav. 79, 377-382.
Chirumbolo, S. (2011). Gelsemine and Gelsemium sempervirens L. extracts in animal behavioral test: comments and related biases. Front. Neurol. 2:31. doi: 10.3389/fneur.2011.00031

Dutt, V., Dhar, V. J., and Sharma, A. (2010). Antianxiety activity of Gelsemium sempervirens. Pharm. Biol. 48, 1091-1096.

Lai, C. K., and Chan, Y. W. (2009). Confirmation of Gelsemium poisoning by targeted analysis of toxic Gelsemium alkaloids in urine. J. Anal. Toxicol. 33, 56-61.

Magnani, P., Conforti, A., Zanolin, E., Marzotto, M., and Bellavite, P. (2010). Dose-effect study of Gelsemium sempervirens in high dilutions on anxiety-related responses in mice. Psychopharmacology (Berl.) 210, 533-545.

Prut, L., and Belzung, C. (2003). The open field as a paradigm to measure the effects of drugs on anxiety-like behaviors: a review. Eur. J. Pharmacol. 463, 3-33.

Venard, C., Boujedaini, N., Belon, P., Mensah-Nyagan, A. G., and Patte-Mensah, C. (2008). Regulation of neurosteroid allopregnanolone biosynthesis in the rat spinal cord by glycine and the alkaloidal analogs strychnine and gelsemine. Neuroscience 153, 154-161.

Venard, C., Boujedaini, N., Mensah-Nyagan, A. G., and Patte-Mensah, C. (2009). Comparative analysis of gelsemine and Gelsemium sempervirens activity on neurosteroid allopregnanolone formation in the spinal cord and limbic system. Evid. Based Complem. Alternat. Med. 2009, 1-9. doi: 10.1093/ecam/nep083

Received: 18 June 2011; accepted: 16 August 2011; published online: 12 September 2011.

Citation: Bellavite P (2011) Gelsemium sempervirens and animal behavioral models. Front. Neur. 2:56. doi: 10.3389/ fneur.2011.00056

This articlewassubmitted to Frontiers in Neuropharmacology, a specialty of Frontiers in Neurology.

Copyright (c) 2011 Bellavite. This is an open-access article subject to a non-exclusive license between the authors and Frontiers Media SA, which permits use, distribution and reproduction in other forums, provided the original authors and source are credited and other Frontiers conditions are complied with. 